\title{
Letter From the Guest Editor
}

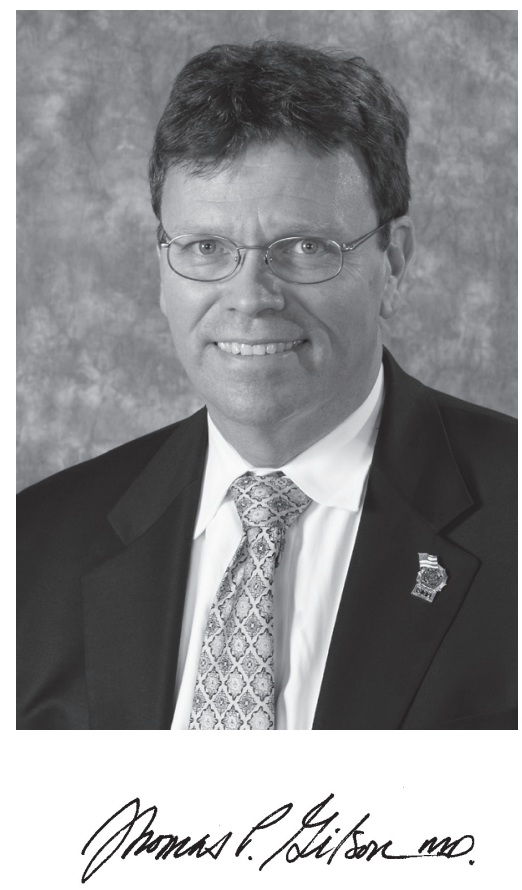

Thomas P. Gilson MD Guest Editor

I am honored to serve as guest editor for this issue of AFP devoted to the opioid crisis. The invited contributors, reviewers, Editor-in-Chief, and staff at $A F P$ have my sincerest thanks for their efforts to get these papers written and submitted, reviewed, edited, and published. This topic is timely both on a public and professional level and the papers cover several areas relevant to the epidemic we are seeing unfold in communities around the nation.

The fundamental aspects of scene investigation are addressed as well as issues around postmortem examination, including the feasibility of compliance with our professional organization's recommendation to conduct full autopsy examinations on drug-related deaths. In many jurisdictions the opioid epidemic has exacerbated the shortcomings of death investigation in the United States both in areas of long-standing de- ficiencies in adequately trained personnel and funding. The National Association of Medical Examiners has long emphasized the need for more forensic pathologists and the increased caseloads precipitated by the opioid crisis are driving this point home in many regions, where several offices now face challenges to maintain adequate staff for accreditation purposes and comply with best practices. These increased caseloads of course ripple into several other areas of forensic practice, most notably toxicology.

Medical examiners are a presence in both legal and public health systems and it is my hope that this issue of $A F P$ addresses both dimensions. As prevention of drug-related deaths becomes an increasing focus of the general community, legal prosecutions have increased and medical testimony is expected. Understanding the legal standards for prosecution (and their 
limitations) creates a climate in which both accountability and fairness can be achieved. Several articles are also devoted to attempts to work within the public health system to impact the drug problem, whether these include using tools like prescription monitoring programs to augment practice or tabulating and sharing data to bring emerging trends in these deaths to attention more rapidly than traditional aggregate death certificate data analysis.
Drug overdose has been the most common form of accidental death for nearly a decade now (surpassing motor vehicle crashes). Tens of thousands of our fellow citizens are dying each year from drug overdoses and these deaths touch many families and friends of the decedents. Listening to the lessons these decedents have to teach is essential in our mission to serve the living. 